\title{
Status epilepticus-induced changes in astrocytic Kir4.1 channel expression in pilocarpine model of temporal lobe epilepsy
}

\author{
Higor A. Iha ${ }^{1}$, Takahiro Mukai ${ }^{1}$, Haruna Imaura ${ }^{1}$, Takayuki Nakamura ${ }^{1}$, Satoko Yamashita ${ }^{1}$, \\ Tatsuya Yamamoto ${ }^{1}$, Saki Shimizu ${ }^{1}$, Kazuaki Sato ${ }^{1,2}$, Akio Ikeda ${ }^{2}$, Yukihiro Ohno ${ }^{1}$ \\ ${ }^{1}$ Laboratory of Pharmacology, Osaka University of Pharmaceutical Sciences, Japan, ${ }^{2}$ Department of Epilepsy, \\ Movement Disorders and Physiology, Kyoto University, Japan
}

Background: Astrocytic inwardly rectifying potassium (Kir) 4.1 channels are responsible for spatial $\mathrm{K}^{+}$buffering in synaptic clefts by removing excessive $\mathrm{K}^{+}$, which consequently controls neural excitability. To evaluate the modulatory role of Kir4.1 channels in the development of temporal lobe epilepsy (TLE), we assessed the expressional changes in astrocytic Kir4.1 channels following pilocarpine (PILO)-induced status epileptics (SE) in PILO-TLE rats.

Methods: Rats were treated with methyl-scopolamine (3 mg/kg, i.p.) and, $30 \mathrm{~min}$ later, injected with PILO (450 mg/kg, i. p.) to induce SE. Animals presenting SE within 15 min were used as SE group and those of non-SE as control. Brain samples were obtained 1, 3 and 7 day(s) after the PILO-induced SE and subjected to immunostaining for Kir4.1 and GFAP (an astrocyte marker). In addition, animals from the group of 3 days were subjected to the seizure sensitivity test using increasing doses of PILO.

Results: GFAP- and Kir4.1-positive astrocytes were significantly reduced by SE in the lateral temporal lobe (i.e., periectorhinal cortex) at day 1 after the PILO-induced SE. However, the numbers of GFAP- and Kir4.1-positive astrocytes in other brain regions (e.g., motor cortex, sensory cortex, hippocampus and amygdala) were unaltered at this stage. At day 3 after the PILO-induced SE, GFAP and Kir4.1 expressions were significantly increased by SE in most regions of the cerebral cortex and amygdala while GFAP- and Kir4.1-positive astrocytes in the lateral temporal lobe were still reduced. The SE-induced changes in GFAP- and Kir4.1-positive astrocytes in each brain regions persisted up to day 7 after the PILO-induced SE. In addition, the SE group animals showed increased susceptibility to PILO seizure induction at day 3 after PILO-induced SE.

Conclusions: The present results showed that PILO-induced SE region-specifically reduces astrocytic Kir4.1 expression in the lateral temporal lobe during the early stage prior to the development of astrogliosis. Relationships in changes between seizure susceptibilities and astrocytic Kir4.1 expression are currently under investigation. 